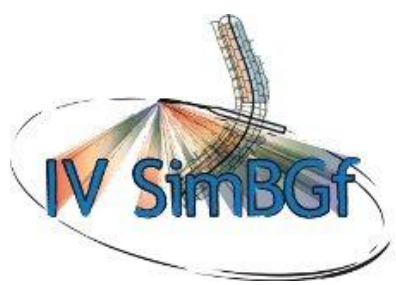

\section{Simulando o tensor de gravidade para melhorar o entendimento das possíveis caracterizações e implicações na estrutura de densidades das bacias sedimentares do Solimões, Amazonas e Parnaíba}

E. P. Bomfim* e E. C. Molina, Instituto de Astronomia, Geofísica e Ciências atmosférica - IAG. Universidade de São Paulo.

Copyright 2010, SBGf - Sociedade Brasileira de Geofísica

Este texto foi preparado para a apresentação no IV Simpósio Brasileiro de Geofísica, Brasília, 14 a 17 de novembro de 2010. Seu conteúdo foi revisado pelo Comitê Técnico do IV SimBGf, mas não necessariamente representa a opinião da SBGf ou de seus associados. É proibida a reprodução total ou parcial deste material para propósitos comerciais sem prévia autorização da SBGf.

\section{Abstract}

The most direct way to detect density anomalies is the study of the gravitational potential and its derivatives. Therefore a study was made from the elements of the gravity field, important quantities that is relating directly to the potential anomaly and deflection of vertical components from recent geopotential models available with regard to a brief description and interpretation of these elements of gravity field. Furthermore, estimates will be obtained of the geoid, the distribution of the gravity anomaly and the components of tensor gradient gravimetric, quantities important for the modeling and study of structures of the Solimões, Amazon and Parnaiba Basin simulating one of the goals of the mission GOCE, the gradiometry gravimetric.

\section{Introdução}

A missão GOCE (Gravity Field and Stead-State Ocean Circulation Explorer) (ESA, 1996) combina o conceito de acelerômetros tridimensionais à bordo com o rastreamento de satélite para obter a anomalia gravimétrica e os componentes do tensor gravimétrico.

À medida que as densidades do manto subjacente parecem exercer controles importantes na formação das bacias, o estudo do campo da gravidade, potencial da gravidade, tensor gradiente e o estado isostático é uma forma essencial para classificá-las. Pesquisas bibliográficas em bacias de larga escala indicam que estas são associadas com crátons nas bordas das bacias ou subjacentes às bacias. Entretanto, devido às grandes espessuras típicas dos sedimentos (4 a $20 \mathrm{~km}$ ), em alguns casos, há ambigüidade se a bacia encontra-se jazida sob um cráton ou não (Braitenberg \& Ebbing, 2007).

Portanto, em virtude da disponibilidade dos seus dados e sua boa resolução, as estimativas do campo da gravidade aqui utilizadas foram feitas a partir dos dados da Missão

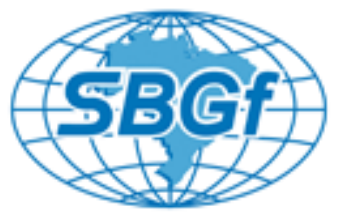

GRACE (Gravity Recovery And Climate Experiment) do modelo do campo da gravidade global combinado EIGENGL04C (Förste et al., 2006) e do modelo geopotencial EGM2008 (Pavlis et al., 2008)

\section{Área de estudo}

A área de estudo concentra-se nas três maiores bacias paleozóicas amazônicas de grande escala no Brasil: a Bacia do Solimões, a Bacia do Amazonas e a Bacia do Parnaíba, que possuem suas expressões situadas entre as coordenadas com longitudes $75^{\circ} \mathrm{W}$ e $40^{\circ} \mathrm{W}$ e latitudes $12^{\circ} \mathrm{S}$ e $5^{\circ} \mathrm{N}$ (Figura 1$)$.

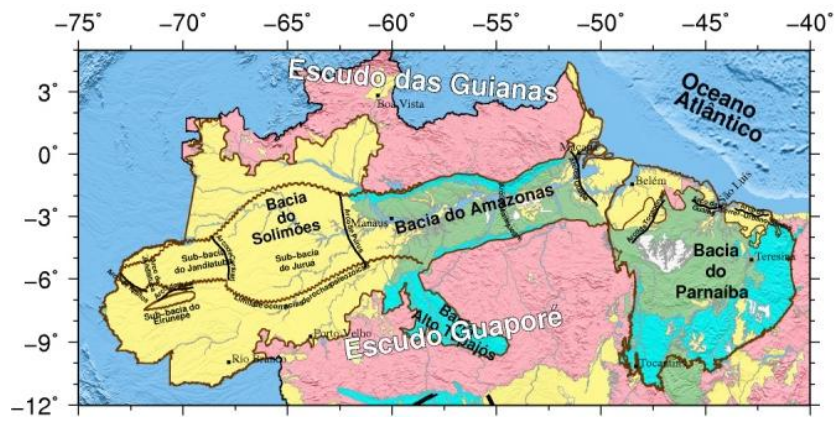

Figura 1: Bacias Paleozóicas Amazônicas - Bacia do Solimões, Amazonas e Parnaíba.

\section{Metodologia}

Nos anos de 1960 a 1980 satélites geodésicos forneceram os componentes do campo da gravidade de longo a médio comprimento de onda através de modelos geopotenciais globais que são representados em harmônicos esféricos. As mais recentes missões de gravimetria por satélite CHAMP (CHAllenging Minisatellite Payload) (Reigber et al., 2002) e GRACE (Tapley et al., 2005) conduziram melhoras significantes a cerca do conhecimento do longo comprimento de onda do campo da gravidade da Terra, e em conseqüência o longo comprimento de onda do geóide. Essas missões forneceram uma cobertura a cerca da informação do campo da gravidade homogênea e quase completa por todo o globo, uma vez que suas órbitas foram aproximadamente polares. No entanto, freqüentemente as estimativas de erro para os modelos geopotenciais são demasiadamente otimistas e/ou apresentam-se como médias globais e, portanto, não representa necessariamente o desempenho dos modelos geopotenciais em uma região particular (Kiamehr \& Eshagh 2008).

Parâmetros do campo da gravidade a partir de modelos geopotenciais globais

O potencial anômalo pode ser utilizado para obter quantidades muito importantes que relaciona o quanto 0 geopotencial difere do esferopotencial e pode ser considerado como o potencial produzido pelas massas anô- 
malas terrestres. Quantidades do campo da gravidade como a altura geoidal, a anomalia da gravidade e os componentes do desvio da vertical são as principais grandezas, as quais podem expressar o desvio do campo normal em relação ao campo real e ser interpretados geofísicamente.

Outra grandeza útil do campo da gravidade é o gradiente da gravidade que é referenciado ao potencial anômalo.

Para análises do campo da gravidade, o potencial anômalo $(T)$ em um ponto qualquer pode ser representado por uma série harmônica esférica (Heiskanen \& Moritz, 1967):

$$
\begin{array}{r}
\mathrm{T}(r, \varphi, \lambda)=\frac{G M}{a} \sum_{l=2}^{L} \sum_{m=0}^{l}\left(\frac{a}{r}\right)^{n+1}\left[\bar{C}_{l m} \cos m \lambda\right. \\
\left.+\overline{\bar{S}}_{l m} \sin m \lambda\right] \bar{P}_{l m}(\cos \varphi)
\end{array}
$$

onde $r, \varphi, \lambda$ são a distância geocêntrica, latitude e longitude geodésicas respectivamente, GM é produto da constante gravitacional pela massa da Terra, a é o semieixo maior do elipsóide de referência, $L$ é o grau máximo, I, $m$ são grau e ordem do harmônico esférico, $\bar{C}_{l m}$ e $\bar{S}_{l m}$ são os coeficientes geopotenciais normalizados, e $\bar{P}_{l m}(\cos \varphi)$ é função de Legendre associada normalizada. De acordo com a fórmula de Bruns (Heiskanen \& Moritz, 1967), temse que

$$
\begin{array}{r}
\mathrm{N}(r, \varphi, \lambda)=\frac{G M}{a \gamma} \sum_{l=2}^{L} \sum_{m=0}^{l}\left(\frac{a}{r}\right)^{n+1}\left[\bar{C}_{l m} \cos m \lambda\right. \\
\left.+\overline{\bar{S}}_{l m} \sin m \lambda\right] \bar{P}_{l m}(\sin \varphi)
\end{array}
$$

onde $\gamma$ é a gravidade normal obtida na latitude do ponto pela fórmula Somigliana. De acordo com Heiskanen \& Moritz (1967), pode-se escrever a anomalia da gravidade como uma função dos coeficientes do geopotencial:

$$
\begin{gathered}
\Delta \mathrm{g}(r, \varphi, \lambda)=\frac{G M}{a} \sum_{l=2}^{L} \sum_{m=0}^{l}\left(\frac{a}{r}\right)^{n+1}\left(\frac{n-1}{r}\right)\left[\bar{C}_{l m} \cos m \lambda\right. \\
\left.+\bar{S}_{l m} \sin m \lambda\right] \bar{P}_{l m}(\sin \varphi)
\end{gathered}
$$

O desvio da vertical é o ângulo formado entre o vetor gravidade ou linha de prumo e o correspondente vetor da gravidade normal ao elipsóide sobre o mesmo ponto. $O$ desvio da vertical tem dois componentes: o componente meridiana $(\eta)$ e o componente primeiro vertical $(\xi)$ e de acordo com Heiskanen \& Moritz (1967) os componentes do desvio da vertical podem ser representados como:

$$
\begin{gathered}
\mathbf{n}(r, \varphi, \lambda)=\frac{G M}{a \gamma r \sin \varphi} \sum_{l=2}^{L} \sum_{m=0}^{l}\left(\frac{a}{r}\right)^{n+1} m\left[\bar{C}_{l m} \cos m \lambda\right. \\
\left.+\bar{S}_{l m} \sin m \lambda\right] \bar{P}_{l m}(\sin \varphi)
\end{gathered}
$$

$\boldsymbol{\xi}(r, \varphi, \lambda)$

$$
\begin{aligned}
& =\frac{G M}{a r \gamma^{2}} \sum_{l=2}^{L} \sum_{m=0}^{l}\left(\frac{a}{r}\right)^{n+1}\left[\bar{C}_{l m} \cos m \lambda\right. \\
& \left.+\bar{S}_{l m} \sin m \lambda\right]\left[\gamma \sqrt{(l-m)(l+m-1)\left(1+\delta_{m}\right)} \bar{P}_{l, m+1}(\sin \varphi)\right. \\
& \left.-\left(\gamma m \tan \varphi-\frac{d \gamma}{d \varphi}\right) \bar{P}_{l m}(\sin \varphi)\right] \\
& \text { onde, }
\end{aligned}
$$

$\frac{d \gamma}{d \varphi}=\frac{\gamma_{0} \cos \varphi \sin \varphi}{\left(1-e^{2} \sin ^{2} \varphi\right)^{\frac{3}{2}}}\left(2 k+e^{2}\left(1-k \sin ^{2} \varphi\right)\right)$

e $\gamma_{0}$ é a gravidade normal no equador, $e^{2}$ é a primeira excentricidade do elipsóide de referência, $k$ é a constante da fórmula e $k=0.00193185353$ baseado na fórmula Somigliana, e $\delta$ é o delta de Kronecker.

Gradiometria: componentes do gradiente da gravidade

Aqui serão utilizadas expressões não singulares para gradientes da gravidade no sistema de referência local orientado para o norte apresentado por Petrovskaya \& Vershkov (2006). A primeira e segunda ordem das derivadas do potencial perturbador $T$ no sistema de referência orientado para 0 norte $\{x, y, z\}$ são consideradas, procedendo-se a partir de uma relação conhecida (Reed, 1973), porém apresentada em termos da latitude ao invés da co-latitude. Assim, a relação entre os componentes das coordenadas cartesianas no sistema local e das coordenadas esféricas geocêntricas torna-se:

$$
\begin{gathered}
T_{z z}=T_{r r}, \\
T_{x x}=\frac{1}{r} T_{r}+\frac{1}{r^{2}} T_{\varphi \varphi}, \\
T_{y y}=\frac{1}{r} T_{r}+\frac{1}{r^{2} \cot \varphi} T_{\varphi}+\frac{1}{r^{2} \cos ^{2} \varphi} T_{\lambda \lambda}, \\
T_{x y}=\frac{1}{r^{2} \cos \varphi} T_{\varphi \lambda}-\frac{\sin \varphi}{r^{2} \cos ^{2} \varphi} T_{\lambda}, \\
T_{\mathrm{xz}}=\frac{1}{r^{2}} T_{\varphi}-\frac{1}{r} T_{r \varphi}, \\
T_{y z}=\frac{1}{r^{2} \cos \varphi} T_{\lambda}-\frac{1}{r \cos \varphi} T_{\mathrm{r} \lambda}
\end{gathered}
$$

Os parâmetros do gradiente da gravidade são apresentados de uma forma alternativa de Petrovskaya \& Vershkov (2006) e suas relações seguem em Kiamehr et al. (2008):

$$
\begin{aligned}
& T_{z z}=\frac{G M}{a^{3}} \sum_{l=2}^{L} \sum_{m=0}^{l}(l+1)(l \\
& +2)\left(\frac{a}{r}\right)^{l+3}\left[\begin{array}{c}
\bar{C}_{l m} \cos m \lambda \\
+\bar{S}_{l m} \sin m \lambda
\end{array}\right] \bar{P}_{l m}(\sin \varphi) \\
& T_{x x}=\frac{G M}{a^{3}} \sum_{l=2}^{L} \sum_{m=0}^{l}\left(\frac{a}{r}\right)^{l+3}\left[\bar{C}_{l m} \cos m \lambda\right. \\
& \left.+\bar{S}_{l m} \sin m \lambda\right]\left\{a_{l m} \bar{P}_{l m-2}(\sin \varphi)\right. \\
& +\left[b_{l m}-(l+1)(l+2)\right] \bar{P}_{l m}(\sin \varphi) \\
& \left.+c_{l m} \bar{P}_{l m+2}(\sin \varphi)\right\} \\
& T_{y y}=-\frac{G M}{a^{3}} \sum_{l=2}^{L} \sum_{m=0}^{l}\left(\frac{a}{r}\right)^{l+3}\left[\begin{array}{c}
\bar{C}_{l m} \cos m \lambda+ \\
\bar{S}_{l m} \sin m \lambda
\end{array}\right]\left\{a_{l m} \bar{P}_{l m-2}(\sin \varphi)\right. \\
& \left.+b_{l m} \bar{P}_{l m}(\sin \varphi)+c_{l m} \bar{P}_{l m+2}(\sin \varphi)\right\}
\end{aligned}
$$

e

$T_{x y}$

$=\frac{G M}{a^{3}} \sum_{l=2}^{L} \sum_{m=1}^{l}\left(\frac{a}{r}\right)^{l+3}\left[\begin{array}{c}\bar{C}_{l m} \sin m \lambda- \\ \bar{S}_{l m} \cos m \lambda\end{array}\right]\left\{\begin{array}{c}d_{l m} \bar{P}_{l-1, m-2}(\sin \varphi)+ \\ g_{l m} \bar{P}_{l-1, m}(\sin \varphi)+ \\ \bar{P}_{l-1, m+2}(\sin \varphi)\end{array}\right\}$ 
$T_{y z}$

$=\frac{G M}{a^{3}} \sum_{l=2}^{L} \sum_{m=1}^{l}\left(\frac{a}{r}\right)^{l+3}\left[\begin{array}{c}\bar{C}_{l m} \sin m \lambda- \\ \bar{S}_{l m} \cos m \lambda\end{array}\right]\left\{\begin{array}{c}\mu_{l m} \bar{P}_{l-1, m-1}(\sin \varphi)+ \\ v_{l m} \bar{P}_{l-1, m+1}(\sin \varphi)\end{array}\right\}$

$T_{x y}=\frac{G M}{a^{3}} \sum_{l=2}^{L} \sum_{m=1}^{l}\left(\frac{a}{r}\right)^{l+3}\left[\begin{array}{c}\left.\bar{C}_{l m} \cos m \lambda+\right] \\ \bar{S}_{l m} \sin m \lambda\end{array}\right]\left\{\begin{array}{c}\beta_{l m} \bar{P}_{l, m-1}(\sin \varphi)+ \\ \gamma_{l m} \bar{P}_{l, m+1}(\sin \varphi)\end{array}\right\}$

Os coeficientes das funções de Legendre são constantes numéricas dadas por Petrovskaya \& Vershkov (2006).

$O$ uso dos dados do gradiente da gravidade (GG) em exploração está se tornando cada vez mais comum. Entretanto, a interpretação destes dados não é tão facilmente familiar quanto os dados da gravidade na vertical. Assim, várias combinações dos componentes do gradiente da gravidade podem ser utilizadas para simplificar seu padrão complexo e adicionar uma melhora, e, portanto, ajudar na interpretação dos dados (Saad, 2006).

Amplitude do gradiente horizontal da gravidade $\left(g_{z}\right)$ pode ser obtida pelos componentes dos tensores dada por:

$$
h_{g}=\sqrt{T_{z x}^{2}+T_{z y}^{2}}
$$

Amplitude do gradiente total ou amplitude do sinal analítico de $\mathrm{g}_{\mathrm{z}}$ é dada por:

$$
A_{S A}=\sqrt{T_{z x}^{2}+T_{z y}^{2}+T_{z Z}^{2}}
$$

Curvatura diferencial é dada por:

$$
C_{g}=\sqrt{\left(T_{x x}-T_{y y}\right)^{2}+\left(2 T_{x y}\right)^{2}}
$$

Os três exemplos dos produtos GG combinados proporcionam mais realces para a parte de alta freqüência das anomalias devido às fontes rasas e produz coordenada independente ou anomalias invariantes. Estes, talvez sejam mais fáceis de se interpretar que os componentes originais do gradiente. Outras coordenadas independentes invariantes podem ser obtidas e usadas igualmente para interpretar os dados utilizando diferentes combinações dos componentes GG.

\section{Resultados preliminares e discussão}

As ondulações do geóide foram reduzidas das variações dos mais longos comprimentos de ondas. Sistematicamente foi subtraído o campo geoidal até grau e ordem 10 na expansão dos harmônicos esféricos (Fig. 2) a fim de obter um campo representativo das estruturas da crosta e manto superior, anomalia Bouguer (Fig. 3a). Esta redução corresponde a subtrair os componentes do campo com comprimento de onda maior que $2000 \mathrm{~km}$ em médias latitudes. O geóide corrigido do terreno, topografia, é análogo ao campo da gravidade Bouguer e pode ser obtido a partir da redução do efeito de massas topográficas pelo cálculo direto de prismas deduzidos a partir de modelo digital de terreno, por exemplo, o modelo de terreno digital Forsberg (1984).
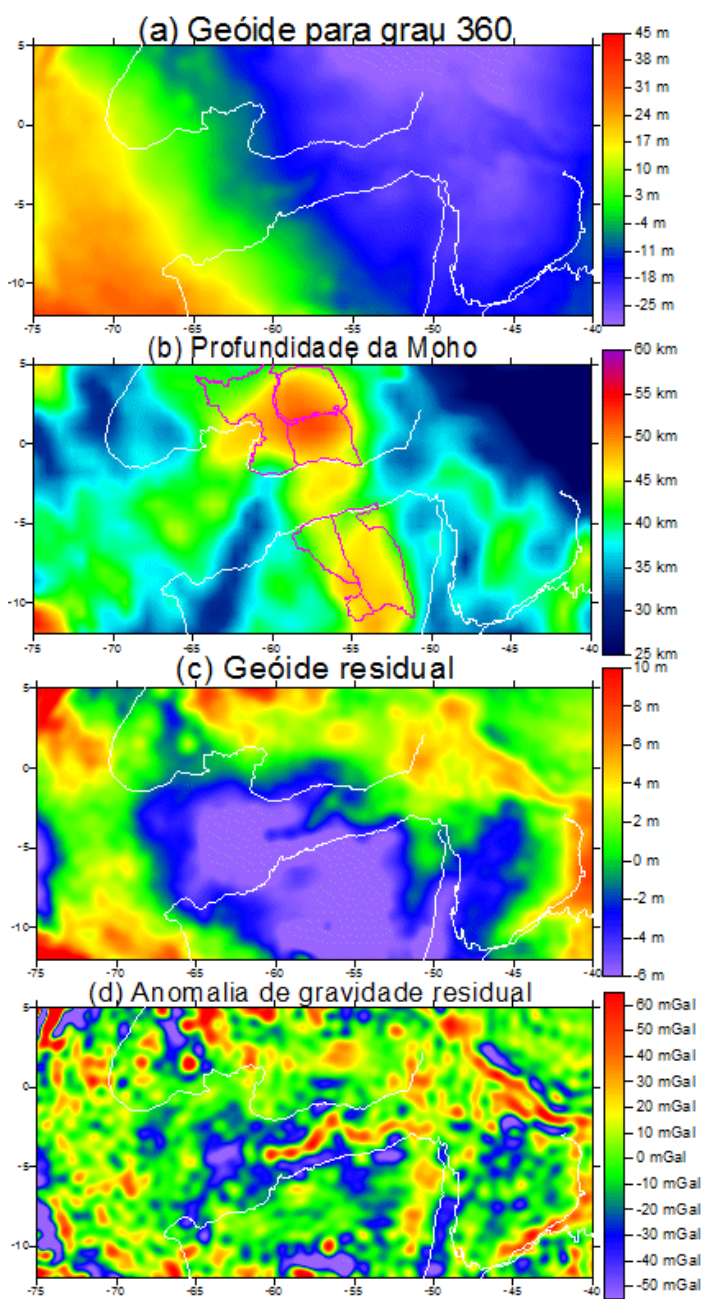

Figura 2: (a) Geóide (m) para o grau e ordem 360; (b) Mapa da profundidade do Moho (espessura crustal); (c) geóide residual $(\mathrm{m})$ e (d) anomalia ar-livre (mGal) residual para as bacias do Solimões, Amazonas e Parnaíba. Dados: EIGEN-GL04C (Förste et al., 2006).

A anomalia Bouguer representa principalmente fontes crustais e constitui um esboço para as variações da espessura da crosta em áreas tectônicas mais jovens. No caso de áreas cratônicas, a base da crosta mostra pouca variação e as características da anomalia Bouguer são principalmente as variações de densidade na crosta ou manto superior.

Na Fig. 3 é mostrado o campo da gravidade Bouguer sobre uma área de aproximadamente $3000 \mathrm{~km}$ de comprimento por $750 \mathrm{~km}$ de largura com sedimentos alcançando $5000 \mathrm{~m}$ nas Bacias do Solimões e Amazonas e 3400 m na Bacia do Parnaíba (Bizzi et al., 2004).

O mapa de anomalia Bouguer mostra uma cadeia de alta gravidade de $+30 \mathrm{a}+60$ mGal que atravessa a bacia coincidindo aproximadamente com as espessuras máximas da rocha sedimentar e portanto esboça a parte mais central (a calha) da Bacia do Amazonas. Os altos da gravidade estão flanqueados pelas baixas gravidades de $40 \pm 20 \mathrm{mGal}$ coincidindo com região de maior espessura 
da crosta, descontinuidade crosta/manto ou profundidade do Moho, obtido por Lloyd et al. (2007) (Fig. 2b). Os valores relativamente altos da anomalia Bouguer em correspondência com a bacia apontam para um afinamento crustal ou adensamento da crosta ou manto superior. Devido aos sedimentos contribuírem para baixar o sinal da gravidade Bouguer, caso nenhum baixo seja observado, deve haver ou um Moho mais rasa ou então um adensamento para balancear o sinal negativo produzido pelos sedimentos.

(a) Anomalia Bouguer

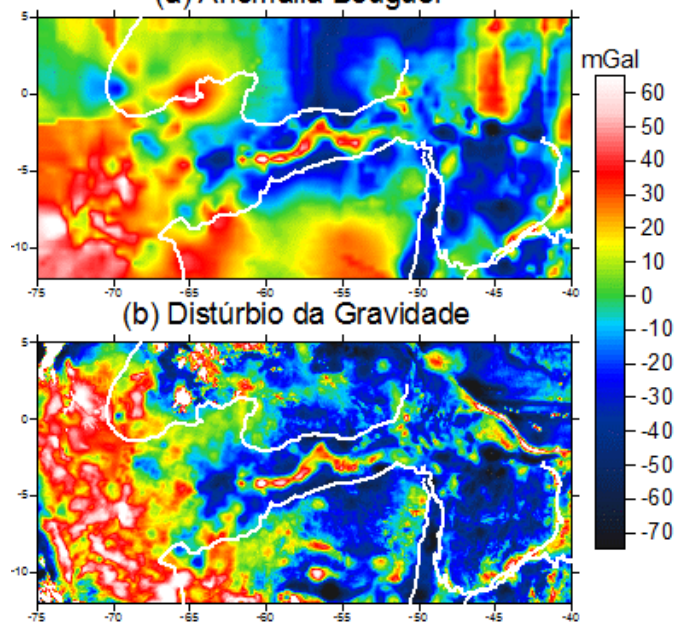

(c) Componente primeiro vertical

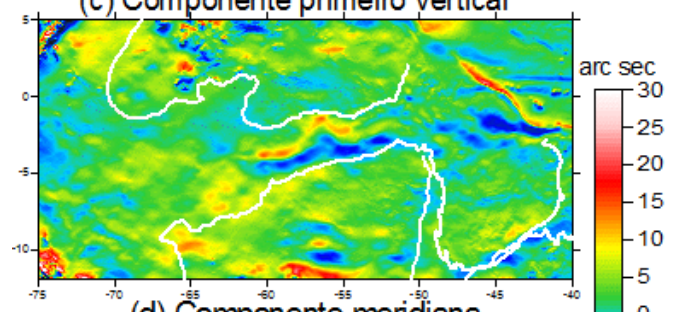

(d) Componente meridiana

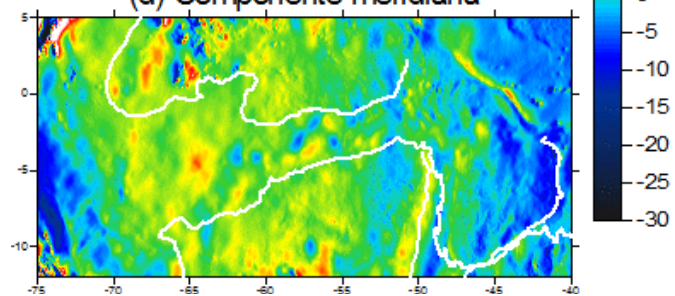

Figura 3: (a) Anomalia Bouguer (mGal) para as bacias do Solimões, Amazonas e Parnaíba. (b) Distúrbio da gravidade; Desvio da vertical nos componentes (c) Componente primeiro da vertical e (d) Componente meridiana. Dados:EGM2008 (Pavlis et al., 2008).

$\mathrm{Na}$ Fig. 3b, observa-se que a boa resolução do modelo EGM2008 (Pavlis et al., 2008) calculado no desenvolvimento dos harmônicos esféricos para o grau e ordem de 2160, faz com que o distúrbio da gravidade forneça um mapa muito mais detalhado, porém parecido com o da anomalia da gravidade, pois o distúrbio da gravidade é grandeza diretamente vinculada ao potencial perturbador e a anomalia da gravidade é expressa como uma combinação linear entre o potencial perturbador e sua derivada normal.
Nos mapas dos componentes do desvio da vertical (Figs. $3 c$ e $3 d$ ), observam-se variações na direção norte-sul e leste-oeste de heterogeneidades no interior da Terra. Tal como vistos nos outros mapas, as espessuras dos sedimentos das bacias do Amazonas e Solimões são novamente caracterizadas nestes componentes. Verifica-se que na Província do Parnaíba é possível identificar no mapa do componente primeiro vertical, que mostra o componente leste-oeste, as divisões das bacias, que coincidem com os limites que dividem as rochas sedimentares da Bacia do Parnaíba: Bacia do Parnaíba propriamente dita, Bacia das Alpercatas, Bacia do Grajaú e Bacia do Espigão-Mestre conforme a classificação desta Província dada em Bizzi et al. (2004).

No entanto, resultados obtidos por Lloyd et al. (2007) da profundidade de Moho (Fig. 2b) mostram consistência no baixo da gravidade com valores já obtidos para estações na área de estudo. Esses resultados mostram que a crosta é mais fina na direção norte da área de estudo, entretanto, a ausência de mais estações na parte superior do Cráton não permite uma discussão sobre um possível afinamento crustal, não evidenciando se ocorre em direção norte ou apenas sob a bacia intracratônica. A anomalia do Moho é mais fraca na Bacia do Amazonas comparado aos escudos adjacentes. Como a espessura crustal no Cráton Amazonas é maior que $50 \mathrm{~km}$ a leste dos escudos das Guianas aumentando continuamente através da Bacia do Amazonas dentro do escudo Guaporé ou Brasil-central, a alta densidade na crosta inferior ou manto superior pode ser levada em conta.

As bacias do Amazonas e Solimões apresentam uma alta gravidade linear que podem alcançar $+50 \mathrm{mGal}$. A explicação atribuída para este sinal pode ser um suposto rifte extinto (abortado) abaixo das bacias (Nunn \& Aires, 1988). No caso da Bacia do Parnaíba, o geoíde residual revela uma variação em larga escala, mas não exibe uma correlação evidente com a bacia.

As ondulações no geóide (Fig. 2a) para as bacias mostraram maior variabilidade, mas mesmo estando em boa correlação com as bacias analisadas por Braitenberg \& Ebbing (2007), pode ser considerado, neste caso, analisando o mapa do Moho (Fig. 2b), que o efeito das cargas topográficas e sedimentares, e as massas de alta densidade na crosta e no manto parecem contribuir com esta maior variabilidade no geóide.

Um pronunciado baixo no geóide residual foi encontrado, conforme se verifica na Fig. 2c para as bacias aqui estudadas, mostrando uma correlação evidente com a área de maior espessura crustal (Fig. 2b). A área de maior espessura crustal coincide com a provícia Tapajós-Parima e Amazônia Central que se encontra tracejada na cor púrpura da esquerda para direita respectivamente na Fig. 2b.

$\mathrm{Na}$ Fig. 4 seguem todos os cinco componentes independentes usados no processo de interpretação para determinar, por exemplo, o centro de massa $\left(T_{x z}\right.$ e $\left.T_{y z}\right)$, extremidades dos corpos ( $T_{y y}$ e $\left.T_{x x}\right)$ e cantos da anomalia $\left(T_{x y}\right)$. Sendo o $T_{z z}$, o componente vertical que mais de perto se assemelha à gravidade convencional em que a anomalia 
é mostrada na posição espacial correta e é correlacionada mais facilmente com a geologia em subsuperfície.

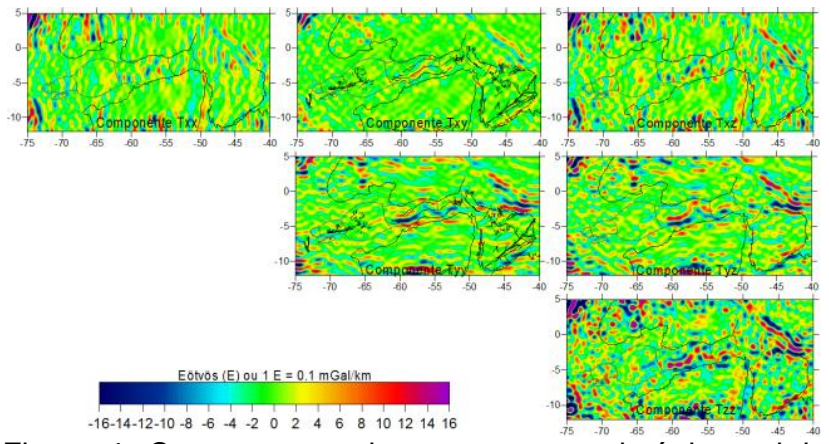

Figura 4: Componentes dos tensores gravimétricos obtidos do modelo geopotencial EIGEN-GL04C (Förste et al., 2006). Os componentes $\mathrm{T}_{\mathrm{xy}}$ e $\mathrm{T}_{\mathrm{yy}}$ foram plotados em conjunto com as principais estruturas da bacia (linha cor preta). Dados da CPRM (Bizzi et al., 2004).

Os cálculos aqui apresentados na Fig. 4, foram obtidos a partir de um campo total fornecido pelo modelo EIGENGL04C para a anomalia da gravidade (anomalia ar-livre), a qual calculou-se as derivadas de cada componente com respeito à posição nas três direções coordenadas. Note que os componentes yx, zx, e zy (não mostrados) são iguais aos componentes $x y, x z$ e $y z$ respectivamente, e que a soma ao longo da diagonal é zero.

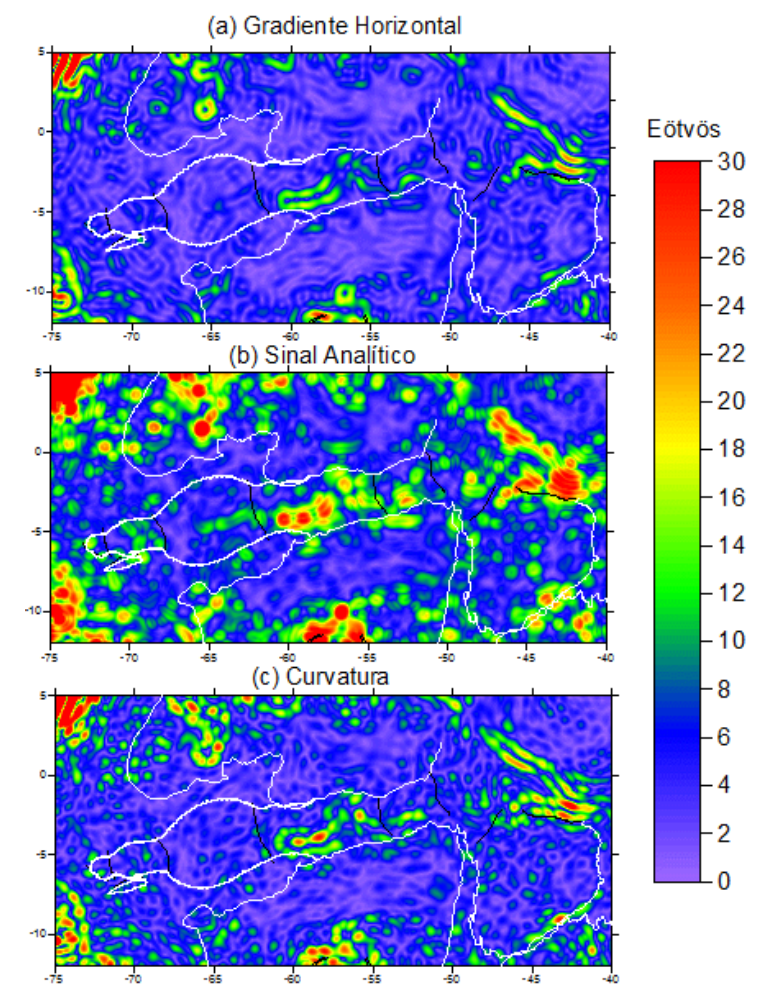

Figura 5: (a) Amplitude do gradiente horizontal da gravidade; (b) amplitude do gradiente total e (c) a curvatura diferencial dos dados obtidos na Fig. 4.

Os componentes $T_{x y}$ e $T_{y y}$ foram plotados em conjunto com as principais estruturas das bacias, arcos, lineamen- tos, calhas, flexura e alto estrutural obtidos dos dados da CPRM descritos em Bizzi et al. (2004), e pela fig. 4, verifica-se que os tensores tiveram uma boa correlação com essas estruturas em subsuperfície. No entanto, quando não se têm alguma informação da geologia em subsuperfície, torna-se bastante moroso e complicado analisar somente os dados individuais dos componentes do gradiente da gravidade.

Portanto, foram feitas combinações dos componentes do gradiente da gravidade para simplificar seu padrão, na maioria das vezes complexo, e adicionar uma melhora, e, portanto, ajudar na interpretação dos dados (Fig. 5).

O gradiente horizontal para a Bacia do Amazonas na fig. 5 a mostra, de fato, uma configuração alongada no sentido ENE-WSW, sendo limitada ao norte e ao sul pelos escudos das Guianas e Brasileiro, respectivamente, a leste pelo Arco de Gurupá, e a oeste pelo Arco de Purus como apontado pelos autores (Nunn \& Aires, 1988; Wanderley Filho, 1991; Wanderley et al., 2005).

\section{Conclusão}

O sinal analítico que auxilia na interpretação de fontes mais profunda parece corroborar com charneiras estruturais no asssoalho da bacia, alinhadas e a grosso modo paralelas às margens norte e sul da mesma, que se separam com menor subsidência relativa às assim chamadas plataformas norte e sul do setor intermediário mais profundo, a calha central, onde o preenchimento sedimentar, em sua maioria de idade paleozóica, atinge valores máximos que podem exceder $5.000 \mathrm{~m}$ como descrito por Cunha et al. (1995). Também pode ser interpretado aqui na magnitude da curvatura (Fig. 5c), que enfatiza fortemente os efeitos das fontes mais rasas associada aos sedimentos e afloramentos da Bacia do Amazonas que se restringem às bordas da bacia, nos locais onde a drenagem corta transversalmente os pacotes rochosos, ou então a algumas pedreiras de lavra de calcários neo-paleozóicos, situadas também nas zonas marginais da bacia.

É possível detectar anomalias gravimétricas positivas nos arcos de lquitos, Purus e Gurupá, bem como no Arco Ferrer-Urbano da Bacia do Parnaíba e o Lineamento Tocantins-Araguaia que limita a Província do Parnaíba na parte oeste com a Província do Tocantins. Entre as grandes feições, destacam-se os arcos de Purus e de Gurupá e as falhas de bordas principalmente na Bacia do Amazonas. O Arco de Purus é um elemento estrutural orientado aproximadamente na direção NW-SE, separando a Bacia do Amazonas da Bacia do Solimões, e pode ser caracterizado nos mapas gravimétricos obtidos pelos modelos geopotenciais. No mapa gravimétrico este arco corresponde a um mínimo Bouguer, indicando que há um espesso pacote sedimentar abaixo dele. Na região do arco, o embasamento da bacia consiste de sedimentos do Proterozóico médio, constitutivos da formação Prosperança. O Arco Gurupá é o elemento estrutural com direção NW-SE que marca a fronteira da Bacia do Amazonas e o graben do Marajó. Essa feição é bem identificada a partir dos elementos do campo da gravidade aqui apresentados na Figs. 2, 3, 4 e 5. 
Este trabalho está aguardando os dados da Missão GOCE que fornecerão os componentes tensores gravimétricos coletados nos seus respectivos eixos ao invés de se derivar os tensores a partir de dados coletados apenas na vertical, como feito aqui.

Espera-se utilizar outros dados tais como as geometrias da espessura de sedimentos, profundidade da descontinuidade de Moho e embasamento conhecidas para as bacias como vínculos para a modelagem 3D da densidade em escala crustal.

\section{Agradecimentos}

Agradeço ao CNPq (Conselho Nacional de Desenvolvimento Científico e Tecnológico) pelo apoio financeiro no desenvolvimento desta pesquisa e ao departamento de Geofísica do IAG/USP por toda sua estrutura no auxílio a esta pesquisa.

\section{Referências}

Bizzi LA, Schobbenhaus C, Vidotti RM, Goncalves JH. 2004. Geologia, Tectônica e Recursos Minerais do Brasil. 1.ed. Brasilia: Editora Universidade de Brasília. v.01. 674p.

Braitenberg C \& Ebbing J. 2007. The Gravity Potential Derivatives as a Means to Classify the Barents Sea Basin in the Context of Cratonic Basins. EGM 2007 International Workshop Innovation in EM, Grav and Mag Methods:a new Perspective for Exploration Capri, Italy, April 15 - 18.

Cunha PRC, Gonzaga FG, Coutinho LFC \& Feijó FJ. 1995. Bacia do Amazonas. Boletin de Geociências da Petrobras, $8(1): 47-55$.

ESA, 1996. Report for Assessment: Gravity Field and Steady-State Ocean Circulation Mission, ESA Publication Division, ESTEC, Noordwijk, The Netherlands, SP-1196(1).

Forsberg R. 1984. A Study of Terrain Reductions, Density Anomalies and Geophysical Inversion Methods in Gravity Field Modelling. Reports of the Department of Geodetic Science and Surveying, No. 355, The Ohio State University, Columbus, Ohio.

Förste C, Flechtner F, Schmidt R, Knig R, Meyer U, Stubenvoll R, Rothacher M, Barthelmes F, Neumayer $H$, Biancale R, Bruinsma S, Lemoine JM, Loyer S. 2006. A mean global gravity field model from the combination of satellite mission and altimetry/gravimetry surface data Eigen-GL04C, Geophysical Research Abstracts, Vol. 8, 03462.

Heiskanen WA and Moritz H. 1967. Physical geodesy. Freeman, San Francisco.

Kiamehr, R and Eshagh, M. 2008. EGMlab, a scientific software for determining the gravity and gradient components from global geopotential models, J. Earth Science Informatics, 1(2): 93-103, Springer (SI).

Kiamehr R, Eshagh M, Sjöberg LE. 2008. Interpretation of general geophysical patterns in Iran based on GRACE gradient component analysis. Acta Geophysica 56(2):440-454

Lloyd S, Van der Lee S, França GS, Assumpção M, Feng M. 2007. New Moho map for South America from receiver functions and surface waves, Eos Trans. AGU,88(23), Jt. Assem. Suppl., Abstrac S44A-04.

Nunn, JA \& Aires JR. 1988. Gravity anomalies and flexure of the lithosphere at the middle Amazon basin, Brazil, J. Geophys. Res., 93, 415-428.

Pavlis NK, Holmes SA, Kenyon SC and Factor JK. 2008. An Earth Gravitational Model to Degree 2160: EGM2008, presented at the 2008 General Assembly of the European Geosciences Union, Vienna, Austria, April 13-18.

Petrovskaya MS and Vershkov AN. 2006. Non-singular expressions for the gravity gradients in the local north-oriented and orbital references frames. J Geod 80:117-127.

Reed GB. 1973. Application of kinematical geodesy for determining the shorts wave length components of the gravity field by satellite gradiometry. Ohio State University, Department of Geodesy, Science, Report No. 201, Columbus, Ohio.

Reigber Ch, Luehr $\mathrm{H}$ and Schwintzer P. 2002. CHAMP Mission Status. Advances in Space Research, Vol. 30, No. 2, pp. 129-134.

Saad AH. 2006. Understanding gravity gradients - a tutorial, Leading Edge, 25, 8, 942-949.

Tapley B, Ries J, Bettadpur S, Chambers D, Cheng M, Condi F, Gunter B, Kang Z, Nagel P, Pastor R, Pekker T, Poole S, Wang F. 2005. GGM02-an improved Earth gravity field model from GRACE. J Geod 79:467-478 doi:10.1007/s00190-005-0480-z

Wanderley Filho JR. 1991. Evolução estrutural da Bacia do Amazonas e sua relação com o embasamento. Belém: Universidade Federal do Pará. 125p. Dissertação (Mestrado).

Wanderley Filho JR, Melo HG, Fonseca VMM, Machado MC. 2005. Bacias sedimentares brasileiras: Bacia do Amazonas. Fundação Paleontológica Phoenix, ano 7, no 82, 6pp. 